\title{
NO ASYMPTOTIC FREE LUNCH REVIEWED IN THE LIGHT OF ORLICZ SPACES
}

\author{
IRENE KLEIN
}

\begin{abstract}
No asymptotic free lunch (NAFL) was introduced in [11] and led to a general version of the Fundamental Theorem of Asset Pricing (FTAP) for large financial markets. The present note observes that NAFL can be defined in a natural way using Orlicz spaces. This gives a transparent proof of the FTAP-result.
\end{abstract}

\section{INTRODUCTION}

In [11] the notion of no asymptotic free lunch (NAFL) was introduced and gave a general version of the Fundamental Theorem of Asset Pricing (FTAP) for large financial markets. The present note is a revision showing that the appropriate theoretical background of the rather 'hand-knitted' proof there is the theory of Orlicz spaces. This new sight adds substantially to the transparency of the FTAP for large financial markets and its proof.

Speaking mathematically, the classical continuous-time model of a financial market is just a stochastic basis $\mathbf{B}=\left(\Omega, \mathcal{F},\left(\mathcal{F}_{t}\right), P\right)$ with a vector-valued semimartingale $S=\left(S_{t}\right)$ interpreted as the price process. The theory initiated by Kabanov and Kramkov in [9] suggests to describe a large financial market by a sequence of classical models $\left(\mathbf{B}^{n}, S^{n}\right)$. In a certain sense this is a modern version of the Huberman-Ross arbitrage pricing theory, see for comparison [20] and [5] and the paper [8] which gives a clear overview of the theory. In [9] it was assumed that for each classical model the set of equivalent martingale measures $M^{n}$ for $S^{n}$ consists of a single measure $Q^{n}$. The authors of [9] introduced the notions of asymptotic arbitrage of first and second kind and showed that their absence is equivalent to the contiguity $\left(P^{n}\right) \triangleleft\left(Q^{n}\right)$ and $\left(Q^{n}\right) \triangleleft\left(P^{n}\right)$, respectively. These results can be understood as one-sided versions of the FTAP for large financial markets as contiguity corresponds to the property of absolute continuity of measures in the classical model. Criteria for the general situation (where $M^{n}$ is not a singleton) look more involved, see [13], [14] and in a different formulation [10]. E.g., in [10] it was shown that the above contiguity conditions can be replaced by $\left(P^{n}\right) \triangleleft\left(\bar{Q}^{n}\right)$ and $\left(\underline{\mathrm{Q}}^{n}\right) \triangleleft\left(P^{n}\right)$, where $\bar{Q}^{n}(A)=\sup _{Q \in M^{n}} Q(A), \underline{\mathrm{Q}}^{n}(A)=\inf _{Q \in M^{n}} Q(A)$.

However, a FTAP in the context of a large financial market might rather be the equivalence of an asymptotic no arbitrage condition and the existence of an equivalent martingale measure for the large financial market, that is, a sequence $Q^{n} \in M^{n}$ such that simultaneously $\left(P^{n}\right) \triangleleft\left(Q^{n}\right)$ and $\left(Q^{n}\right) \triangleleft\left(P^{n}\right)$. In [11] this property of bicontiguity was related to the condition NAFL (no asymptotic free lunch). The

Date: November 14, 2006.

1991 Mathematics Subject Classification. 46A20, 46A22, 46N10, 60G44, 60H05.

Key words and phrases. fundamental theorem of asset pricing, equivalent martingale measure, free lunch, large financial market, asymptotic free lunch, contiguity of measures, Orlicz space. 
idea of NAFL is to generalize the classical no free lunch condition of Kreps [16] for a sequence of market models. The definition of NAFL uses a special description of the zero-neighbourhoods for the Mackey-topology of $L^{\infty}$, which was done in a self-made way that conceals the connection to the theory of Orlicz spaces. The aim of the present note is to reveal this connection and thus present a better looking candidate for NAFL which is formulated using polars of balls of Orlicz spaces. The proof of the FTAP-result is recalled now using this NAFL; it is more transparent as definitions are more natural and some technicalities become superfluous being straightforward consequences of properties of $N$-functions. Moreover in Chapter 5 another version of the NAFL condition is presented, which uses the norm-topology of Orlicz spaces instead of the Mackey-topology. This definition turns out to be equivalent to the original one and is of interest as the approximation in the nonmetrizable Mackey-topology is replaced by an approximation with respect to norms. The connection to the no free lunch condition of Kreps becomes clear and is based on a characterization of the weak-star-closure of a convex subset of $L^{\infty}$ in terms of Orlicz spaces, see also [17].

\section{Definitions AND NOtATiOns}

Let $\left(\Omega, \mathcal{F},\left(\mathcal{F}_{t}\right)_{t \in[0, T]}, P\right)$ be a filtered probability space where the filtration satisfies the usual assumptions. (We choose a finite time horizon $T>0$ to avoid technical subtleties, but note that $T=\infty$ works as well.) Whenever it is clear which $P$ is meant, the notation $L^{p}$ is used for $L^{p}(\Omega, \mathcal{F}, P)$; whenever the dependence on a certain measure $R$ is stressed we use the notation $L^{p}(R)$. Let $\left(S_{t}\right)_{t \in[0, T]}$ be an $\left(\mathcal{F}_{t}\right)$-adapted semimartingale with values in $\mathbb{R}^{d}$, describing the price processes of $d$ tradeable assets. Let $H$ be a predictable $S$-integrable process and $(H \cdot S)_{t}$ the stochastic integral of $H$ with respect to $S$. The process $H$ is an admissible trading strategy if there is $a>0$ such that $(H \cdot S) \geq-a$. Define

$$
\mathbf{K}=\left\{(H \cdot S)_{T}: H \text { admissible }\right\} \text { and } \mathbf{C}=\left(\mathbf{K}-L_{+}^{0}\right) \cap L^{\infty} .
$$

$\mathbf{K}$ can be interpreted as the cone of all replicable claims, and $\mathbf{C}$ is the cone of all claims in $L^{\infty}$ that can be superreplicated. Define the set $M_{a}$ of absolutely continuous and the set $M$ of equivalent separating measures

$$
M_{a}=\left\{Q \ll P: E_{Q}[f] \leq 0 \text { for all } f \in \mathbf{K}\right\} \text { and } M=\left\{Q \in M_{a}: Q \sim P\right\} .
$$

If $S$ is bounded (locally bounded) then $M_{a}(M)$ consists of all $P$-absolutely continuous ( $P$-equivalent) probability measures such that $S$ is a martingale (local martingale). In general, for unbounded $S, M_{a}(M)$ is the set of $P$-absolutely continuous ( $P$-equivalent) probabilities such that the admissible stochastic integrals are supermartingales.

Recall two well-known generalizations of the no arbitrage condition, namely, no free lunch with vanishing risk (NFLVR) and no free lunch (NFL):

$$
\begin{array}{cc}
(\mathrm{NFLVR}) & \overline{\mathbf{C}} \cap L_{+}^{\infty}=\{0\} \\
(\mathrm{NFL}) & \overline{\mathbf{C}}^{*} \cap L_{+}^{\infty}=\{0\},
\end{array}
$$

where $\overline{\mathbf{C}}$ is the $L^{\infty}$-norm closure and $\overline{\mathbf{C}}^{*}$ the weak-star-closure of $\mathbf{C}$.

The Fundamental Theorem of Asset Pricing (FTAP) says that an appropriate 'no-arbitrage'-condition is equivalent to $M \neq \emptyset$. Kreps [16] proved that NFL is equivalent to $M \neq \emptyset$. Delbaen and Schachermayer [1] introduced NFLVR and proved the deep theorem that under NFLVR we have $\mathbf{C}=\overline{\mathbf{C}}^{*}$. Thus, the seemingly 
weaker condition NFLVR is, in fact, equivalent to NFL. Moreover, in [2] they showed that, under NFLVR, the set $M^{\sigma}$ of all equivalent sigma-martingale measures is dense in $M$. Therefore NFLVR is equivalent to the existence of an equivalent $\sigma$-martingale measure, compare also [7].

In a large financial market a sequence of market models is considered, that is, a sequence of semimartingales $S^{n}$ based on $\left(\Omega^{n}, \mathcal{F}^{n},\left(\mathcal{F}_{t}^{n}\right), P^{n}\right)$. The interpretation of the superscript $n$ in expressions such as $\mathbf{K}^{n}, \mathbf{C}^{n}, M_{a}^{n}, M^{n}$ etc. is then obvious. Throughout the paper we assume

$$
M^{n} \neq \emptyset, \quad \text { for all } n \in \mathbb{N} .
$$

So, any no arbitrage condition (such as NFLVR and NFL) holds for each model.

However, there is still the possibility of various approximations of an arbitrage profit by trading on the sequence of small markets, compare for example [9], [10], [13], [14]. The present note is focused on the condition no asymptotic free lunch (NAFL) which is the large financial market analogue of NFL, see [11].

The following object plays the role of an equivalent (sigma-, local-) martingale measure for the large financial market: it is a sequence of measures $Q^{n} \in M^{n}$ such that $\left(Q^{n}\right) \triangleleft \triangleright\left(P^{n}\right)$; here the notation $\left(Q^{n}\right) \triangleleft \triangleright\left(P^{n}\right)$ means that the sequence of probability measures $\left(Q^{n}\right)$ is contiguous with respect to the sequence of probability measures $\left(P^{n}\right)$ and vice versa.

Definition 2.1. A sequence of measures $\left(Q^{n}\right)$ is called contiguous with respect to $\left(P^{n}\right)$, denoted by $\left(Q^{n}\right) \triangleleft\left(P^{n}\right)$, if and only if for any sequence $\left(A^{n}\right)_{n=1}^{\infty}, A^{n} \in \mathcal{F}^{n}$, $P^{n}\left(A^{n}\right) \rightarrow 0$ implies that $Q^{n}\left(A^{n}\right) \rightarrow 0$.

Let now $Q^{n} \ll P^{n}$, for all $n$. Throughout the paper we will use the notation $Z^{n}$ for $\frac{d Q^{n}}{d P^{n}}$. Then an alternative criterion to $\left(Q^{n}\right) \triangleleft\left(P^{n}\right)$ is that $\left(Z^{n} \mid Q^{n}\right)$ is $\mathbb{R}$-tight which is equivalent to the condition that $\left(Z^{n} \mid P^{n}\right)$ is uniformly integrable. Compare [6], Lemma V.1.6 and V.1.10, for these criteria in a more general form. Another obvious formulation of contiguity shows that it is a concept of absolute continuity in a uniform way for sequences of probability measures: indeed, $\left(Q^{n}\right) \triangleleft\left(P^{n}\right)$ is equivalent to the following condition: for all $\varepsilon>0$ there is $\delta>0$ such that, for all $n \in \mathbb{N}$ and $A^{n} \in \mathcal{F}^{n}, P^{n}\left(A^{n}\right)<\delta$ implies $Q^{n}\left(A^{n}\right)<\varepsilon$.

\section{NAFL AND THE FTAP FOR LARGE FINANCIAL MARKETS}

A FTAP for large financial markets is a theorem that shows the equivalence between a condition of no asymptotic arbitrage type and the existence of a sequence of measures $Q^{n} \in M^{n}$ such that $\left(Q^{n}\right) \triangleleft \triangleright\left(P^{n}\right)$. In general, one has to use the condition of no asymptotic free lunch (NAFL) of [11] to get this equivalence. A recent result shows that one gets the equivalence with an asymptotic condition of no market free lunch type as well, but this is a slightly different approach using preferences of investors, see [12] and [3]. In the present note we will see that NAFL can be defined in a more elegant way using polars of balls of Orlicz spaces as Mackey-neighbourhoods of 0 in $L^{\infty}$. In the original definition this connection of the Mackey-topology to the theory of Orlicz spaces is concealed as there a rather 'hand-knitted' description of the Mackey-neighbourhoods of 0 was used.

First recall the definition of NAFL. We will define the sets $V^{F, n} \subseteq L^{\infty}\left(P^{n}\right)$ below. In Section 4 we will see that, when $F$ runs through all $N$-functions (see Definition 4.1), these sets form a fundamental system for all Mackey-neighborhoods 
of 0 of $L^{\infty}\left(P^{n}\right)$. Compare [11] for a definition of $V^{F, n}$ that works but is artificial and complicated. For each $\varepsilon>0$ let

$$
D^{\varepsilon, n}=\left\{w \in L^{\infty}\left(P^{n}\right): 0 \leq w \leq 1 \text { and } E_{P^{n}}[w] \geq \varepsilon\right\} .
$$

Remark 3.1. For a sequence $w^{n} \in D^{\alpha, n}$ the following holds: if $\left(P^{n}\right) \triangleleft\left(Q^{n}\right)$ then there is $\beta>0$ such that $w^{n} \in D^{\beta}\left(Q^{n}\right)$ for all $n \in \mathbb{N}$.

Indeed, it is clear that $P^{n}\left(w^{n} \geq \frac{\alpha}{2}\right) \geq \frac{\alpha}{2}$ for each $w^{n} \in D^{\alpha, n}$. By contiguity there is $\delta>0$ such that $Q^{n}\left(w^{n} \geq \frac{\alpha}{2}\right) \geq \delta$, for all $n$, and so $E_{Q^{n}}\left[w^{n}\right] \geq \frac{\alpha}{2} \delta=: \beta$.

Let $F$ be an $N$-function (see Definition 4.1 below). We put

$$
B^{F}\left(P^{n}\right)=\left\{f \in L^{1}\left(P^{n}\right): E_{P^{n}}[F(|f|)] \leq 1\right\} .
$$

Let $V^{F}\left(P^{n}\right)$ be the polar of $B^{F}\left(P^{n}\right)$, that is,

$$
\begin{aligned}
V^{F}\left(P^{n}\right) & =\left(B^{F}\left(P^{n}\right)\right)^{\circ} \\
& =\left\{g \in L^{\infty}\left(P^{n}\right):\left|E_{P^{n}}[g h]\right| \leq 1 \text { for all } h \in B^{F}\left(P^{n}\right)\right\} .
\end{aligned}
$$

We use the notations $B^{F, n}$ and $V^{F, n}$ for $B^{F}\left(P^{n}\right)$ and $V^{F}\left(P^{n}\right)$, respectively, if it is clear which measure $P^{n}$ is meant. In Section 5 we will see the connection to Orlicz spaces and, in particular, that the set $B^{F, n}$ is the closed unit ball of the Orlicz space $L_{F}\left(P^{n}\right)$.

Definition 3.2. We say that the large financial market satisfies the NAFL condition if for any $\varepsilon>0$ there exists an $N$-function $F$ such that, for all $n \in \mathbb{N}$, $\mathbf{C}^{n} \cap\left(D^{\varepsilon, n}+V^{F, n}\right)=\emptyset$.

This means that $\mathbf{C}^{n}$ is, for each $\varepsilon>0$, separated from $D^{\varepsilon, n}$ by some Mackeyneighbourhood $V^{F, n}$ of 0 (where the $F$ does not depend on $n$ ). NAFL is the analogue of NFL for a sequence of $L^{\infty}$ spaces as it is not possible to approximate a strictly positive gain by elements of the sequence of sets $\left(\mathbf{C}^{n}\right)_{n \in \mathbb{N}}$ in a Mackey sense (or, equivalently, as the sets $\mathbf{C}^{n}$ are convex, in a weak star sense). The following version of the FTAP for large financial markets holds, see [11].

Theorem 3.3. NAFL $\Leftrightarrow$ there is $Q^{n} \in M^{n}$ such that $\left(Q^{n}\right) \triangleleft \triangleright\left(P^{n}\right)$.

If $S^{n}$ is (locally) bounded, for all $n$, then $\left(Q^{n}\right)$ is a sequence of (local) martingale measures. For unbounded $S^{n}$, Theorem 3.3 implies the existence of a bicontiguous sequence of sigma-martingale-measures. This is an easy consequence of the fact that $M^{\sigma, n}$ is dense in $M^{n}$ for the variation topology.

\section{N-FUnCtions, NAFL AND the Proof of The FTAP}

One of the two crucial properties of the sets $V^{F}$, for all $F \in \mathcal{N}$, in Definition 3.2 is that they form a fundamental system for all Mackey-neighbourhoods of 0 . The Mackey-topology of $L^{\infty}$ is the topology of uniform convergence on all weakly compact subsets of $L^{1}$, see [4]. A fundamental system for all Mackey-neighbourhoods of 0 is given by the polars of all weakly compact subsets of $L^{1}$. So one should look for an appropriate way to describe all weakly compact subsets of $L^{1}$. To this end we introduce a class of Young functions, the so-called $N$-functions, see [19].

Definition 4.1. $F:[0, \infty) \rightarrow[0, \infty)$ is an $N$-function if $F$ is convex, continuous, $F(0)=0, \frac{F(t)}{t} \uparrow \infty$ as $t \uparrow \infty$ and $\frac{F(t)}{t} \downarrow 0$ as $t \downarrow 0$. The set of all $N$-functions is denoted by $\mathcal{N}$. 
Remark 4.2. Note that in [12] we used a more restrictive (that is differentiable) class of Young functions to define NAFL. However, all results of [12] hold as well when we use the class $\mathcal{N}$ above, as all relevant results of Kusuoka [17], which were referred to, hold for this class as well.

Lemma 4.3. The set $B^{F}$ of (3.2) is closed with respect to $L^{1}$-norm and uniformly integrable. In particular, $\sup _{h \in B^{F}} E\left[|h| \mathbf{1}_{\{|h| \geq \kappa\}}\right] \leq \frac{\kappa}{F(\kappa)}$. On the other hand, for each uniformly integrable $A \subseteq L^{1}$ there is $F \in \mathcal{N}$ such that $A \subseteq B^{F}$.

Proof. The proof of uniform integrability of $B^{F}$ follows by the definition of $B^{F}$ and the properties of the function $F$ (criterion of De La Vallée-Poussin). To get the inequality note that for $h \in B^{F}$ and $\kappa>0$

$$
E\left[|h| \mathbb{1}_{\{|h| \geq \kappa\}}\right]=E\left[\frac{|h|}{F(|h|)} F(|h|) \mathbb{1}_{\{|h| \geq \kappa\}}\right] \leq \frac{\kappa}{F(\kappa)} E[F(|h|)] \leq \frac{\kappa}{F(\kappa)},
$$

as $\frac{F(y)}{y}$ is increasing. To show closedness in $L^{1}$ take $h^{n} \in B^{F}$ with $h^{n} \rightarrow h$ in $L^{1}$, then a subsequence of $F\left(\left|h^{n}\right|\right.$ ) (still denoted by $n$ ) converges to $F(|h|)$ a.s. By Fatou

$$
E[F(|h|)]=E\left[\lim F\left(\left|h^{n}\right|\right)\right] \leq \liminf E\left[F\left(\left|h^{n}\right|\right)\right] \leq 1,
$$

as $F\left(\left|h^{n}\right|\right) \geq 0$. This shows that $h \in B^{F}$. The second part of the statement follows again by De La Vallée-Poussin.

Remark 4.4. $B^{F}$ is a weakly compact convex balanced subset of $L^{1}$. Therefore, by the Bipolar Theorem, $B^{F}=\left(B^{F}\right)^{\circ \circ}=\left(V^{F}\right)^{\circ}$, see [4]. Indeed, it is clear that $B^{F}$ is balanced and convex. So $L^{1}$-closedness implies closedness for the topology $\sigma\left(L^{1}, L^{\infty}\right)$. By the Dunford-Pettis criterion (see for example [21]) a subset of $L^{1}$ is relatively weakly compact if and only if it is uniformly integrable. So, by Lemma 4.3 we get weak compactness of $B^{F}$.

Lemma 4.3 and Remark 4.4 show that the weakly compact subsets of $L^{1}$ can indeed be described completely with the help of the sets $B^{F}$. Hence the polars $V^{F}$ of all $B^{F}$ form a fundamental system of the Mackey 0-neighbourhoods. Moreover, by Remark 4.4 the sets $V^{F}$ fulfill the second crucial property which was used in the proof in [11]. Namely, $\left(V^{F}\right)^{\circ}=B^{F}$ which is uniformly integrable. Later on this will give that $\left(Q^{n}\right) \triangleleft\left(P^{n}\right)$ because of the following relation of contiguity of sequences of measures to the sets $B^{F}$.

Lemma 4.5. Let $Q^{n} \ll P^{n}$, for all $n$. Then $\left(Q^{n}\right) \triangleleft\left(P^{n}\right)$ if and only if there is $F \in \mathcal{N}$ such that, for all $n, Z^{n} \in B^{F, n}$.

Proof. $\left(Q^{n}\right) \triangleleft\left(P^{n}\right)$ if and only if $\left(Z^{n} \mid P^{n}\right)$ is uniformly integrable. The rest follows by Lemma 4.3 , details in [12].

Proof of Theorem 3.3. $(\Rightarrow)$ The proof works exactly as in [11] but for the new sets $V^{F, n}$. For the convenience of the reader we provide the details. By NAFL, for any $\varepsilon>0$, there is $F_{\varepsilon} \in \mathcal{N}$ such that, for all $n, \mathbf{C}^{n} \cap\left(D^{\varepsilon, n}+V^{F_{\varepsilon}, n}\right)=\emptyset$. For notational simplicity we suppress the indices $n$ and $\varepsilon$ for the moment, but keep in mind that everything holds for any $n$ and that $F=F_{\varepsilon}$ and $D=D^{\varepsilon}$. As $V^{F}=-V^{F}$ we have that $\left(\mathbf{C}+V^{F}\right) \cap D=\emptyset$. Replace for the moment $V^{F}$ by its non-empty Mackey-interior $\hat{V}^{F}$, then, by Hahn-Banach, we can separate the disjoint convex 
sets $D$ and $A=\mathbf{C}+\hat{V}^{F}$ (which is Mackey-open). This gives $g \in L^{1},\|g\|_{L^{1}}=1$, such that for the original $V^{F}$ we still have that

$$
\sup _{f \in \mathbf{C}+V^{F}} E[f g] \leq \inf _{h \in D} E[h g] .
$$

As $0 \in V^{F}$ and $-L_{+}^{\infty} \subseteq \mathbf{C}$ we have that $g \geq 0$. As $\mathbf{C}$ is a cone we have that the lefthand side of (4.1) is $\leq 0$. This gives in particular that $g$ is the density of a measure $Q \in M_{a}$. Moreover, $0 \in \mathbf{C}$ and for all $h \in D$ we have that $E[h g] \leq\|h\|_{L^{\infty}}\|g\|_{L^{1}} \leq 1$. Therefore by (4.1) and as $V^{F}=-V^{F}$ we get

$$
\sup _{f \in V^{F}}|E[f g]| \leq 1
$$

hence $g \in\left(V^{F}\right)^{\circ}=B^{F}$.

We claim that there is $\delta$ depending only on $\varepsilon$ (but not on $n$ ) such that

$$
\inf _{h \in D} E[g h] \geq \delta
$$

Indeed, as $g \in B^{F}$, by Lemma 4.3 we have that $E\left[g \mathbb{1}_{\{g \geq \kappa\}}\right] \leq \frac{\kappa}{F(\kappa)}$. Choose $\kappa$ large enough such that $\frac{\kappa}{F(\kappa)}<\frac{1}{3}$. Then it is easy to see (use $E[g]=1$ ) that

$$
P\left(g \geq \frac{1}{3}\right) \geq \frac{1}{3 \kappa} .
$$

Define now $\delta=\frac{1}{9 \kappa \gamma}$, where $\gamma>0$ is a uniform $L^{1}$-bound of the uniformly integrable set $B^{F}$. Note that $\gamma$ and $\kappa$ depend only on $\varepsilon$ and so does $\delta$. Clearly, we have that $\bar{f}:=\frac{1}{\gamma} \mathbb{I}_{\left\{g \geq \frac{1}{3}\right\}} \in V^{F}=\left(B^{F}\right)^{\circ}$. Hence we get

$$
\inf _{h \in D} E[h g] \geq \sup _{f \in V^{F}} E[f g] \geq E[\bar{f} g] \geq \frac{1}{3 \gamma} P\left(g \geq \frac{1}{3}\right) \geq \delta,
$$

where the first inequality holds by (4.1) and the last one by (4.2).

Let us summarize what we proved. For all $\varepsilon>0$ there is $F_{\varepsilon} \in \mathcal{N}, Q^{n, \varepsilon} \in M_{a}^{n}$ and $\delta>0$ such that, for all $n$,

1. $Z^{n, \varepsilon} \in B^{F_{\varepsilon}, n}$ (and so by Lemma $4.4,\left(Q^{n, \varepsilon}\right) \triangleleft\left(P^{n}\right)$, for all $\varepsilon$ ),

2. $P^{n}\left(A^{n}\right) \geq \varepsilon$ implies that $Q^{n, \varepsilon}\left(A^{n}\right) \geq \delta\left(\right.$ as $\left.\mathbb{1}_{A^{n}} \in D^{\varepsilon, n}\right)$.

Let now $\varepsilon=2^{-j}, j \geq 1$, and define $Q^{n}=\sum_{j=1}^{\infty} 2^{-j} Q^{n, 2^{-j}}$. For this sequence it holds that $Q^{n} \in M^{n}$ and $\left(Q^{n}\right) \triangleleft \triangleright\left(P^{n}\right)$, see Lemma 4.6 below.

$(\Leftarrow)$ Suppose that NAFL does not hold. As $\left(Q^{n}\right) \triangleleft\left(P^{n}\right)$ there is $\varphi \in \mathcal{N}$ such that, for all $n, Z^{n} \in B^{\varphi, n}$. Define, for each $\varepsilon>0, F_{\varepsilon} \in \mathcal{N}$ by $F_{\varepsilon}(x):=\varphi(\varepsilon x)$. Observe that $V^{F_{\varepsilon}, n}=\varepsilon V^{\varphi, n}$. (Indeed, it is easy to see that $\frac{1}{\varepsilon} B^{\varphi, n}=B^{F_{\varepsilon}, n}$ and so $\varepsilon\left(B^{\varphi, n}\right)^{\circ}=V^{F_{\varepsilon}, n}$ by the properties of the polar.) By assumption there is $\alpha>0$ such that for $F_{\varepsilon}$ there is $n=n\left(F_{\varepsilon}\right)$ and $f^{\varepsilon} \in \mathbf{C}^{n}$ with $f^{\varepsilon}=h^{\varepsilon}+g^{\varepsilon}$, where $h^{\varepsilon} \in D^{\alpha, n}$ and $g^{\varepsilon} \in V^{F_{\varepsilon}, n}$. As $Q^{n} \in M^{n}$ we have that $E_{Q^{n}}\left[f^{\varepsilon}\right] \leq 0$. Moreover $\left|E_{Q^{n}}\left[g^{\varepsilon}\right]\right|=\left|E_{P^{n}}\left[Z^{n} g^{\varepsilon}\right]\right| \leq \varepsilon$, as $Z^{n} \in B^{\varphi, n}$ and $g^{\varepsilon} \in \varepsilon V^{\varphi, n}$. Hence

$$
E_{Q^{n}}\left[h^{\varepsilon}\right]=E_{Q^{n}}\left[f^{\varepsilon}\right]+E_{Q^{n}}\left[-g^{\varepsilon}\right] \leq \varepsilon .
$$

As $\left(P^{n}\right) \triangleleft\left(Q^{n}\right)$, by Remark 3.1 there is $\beta>0$ such that $\inf _{h \in D^{\alpha, n}} E_{Q^{n}}[h] \geq \beta$ for any $n$. This is a contradiction for small $\varepsilon$.

Lemma 4.6. Suppose that for each $\varepsilon>0$ there is a sequence $Q^{n, \varepsilon} \in M_{a}^{n}$ such that 
i) there is $\delta>0$ such that for all $n$ and $A^{n} \in \mathcal{F}^{n}, P^{n}\left(A^{n}\right) \geq \varepsilon$ implies that $Q^{n, \varepsilon}\left(A^{n}\right) \geq \delta$, and

ii) $\left(Q^{n, \varepsilon}\right) \triangleleft\left(P^{n}\right)$.

Let $Q^{n}=\sum_{j=1}^{\infty} 2^{-j} Q^{n, 2^{-j}}$. Then $Q^{n} \in M^{n}$ and, moreover, $\left(Q^{n}\right) \triangleleft \triangleright\left(P^{n}\right)$.

Proof. $\left\{Z^{n}=0\right\} \subseteq \bigcap_{j=1}^{\infty}\left\{Z^{n, 2^{-j}}<\delta_{j}\right\}$, so i) implies that $P^{n}\left(Z^{n}=0\right)=0$. Hence $Q^{n} \in M^{n}$. Let us now prove that $\left(Q^{n}\right) \triangleleft\left(P^{n}\right)$. Let $\gamma>0$ be arbitrary but fixed. We have to show that there is $\mu>0$ such that $P^{n}\left(A^{n}\right)<\mu$ implies $Q^{n}\left(A^{n}\right)<\gamma$ for all $A^{n} \in \mathcal{F}^{n}$. Let $N \in \mathbb{N}$ be large enough such that $\sum_{j=N+1}^{\infty} 2^{-j}<\frac{\gamma}{2}$. By ii) $\left(Q^{n, 2^{-j}}\right)_{n \geq 1} \triangleleft\left(P^{n}\right)_{n \geq 1}$ for $j=1,2, \ldots, N$, whence for each $j$ there exists $\mu_{j}>0$ such that, for all $n, P^{n}\left(A^{n}\right)<\mu_{j}$ implies that $Q^{n, 2^{-j}}\left(A^{n}\right)<\frac{\gamma}{2}$. Let now $\mu=\min _{j \leq N} \mu_{j}$ and $A^{n} \in \mathcal{F}^{n}$ be such that $P^{n}\left(A^{n}\right)<\mu$. Then

$$
Q^{n}\left(A^{n}\right)=\sum_{j=1}^{N} 2^{-j} Q^{n, 2^{-j}}\left(A^{n}\right)+\sum_{j=N+1}^{\infty} 2^{-j} Q^{n, 2^{-j}}\left(A^{n}\right)<\frac{\gamma}{2}+\frac{\gamma}{2}=\gamma .
$$

To prove that $\left(P^{n}\right) \triangleleft\left(Q^{n}\right)$ observe that i) implies the following: for all $j \in \mathbb{N}$, there is $\mu_{j}$ such, that for any $n, Q^{n, 2^{-j}}\left(A^{n}\right)<\mu_{j}$ implies that $P^{n}\left(A^{n}\right)<2^{-j}$. Let $\gamma>0$ be fixed and choose $N \in \mathbb{N}$ such that $2^{-(N-1)}<\gamma$. Define $\mu=2^{-2 N} \mu_{N}$. Let now $A^{n} \in \mathcal{F}^{n}$ such that $Q^{n}\left(A^{n}\right)<\mu$. Then

$$
\begin{aligned}
P^{n}\left(A^{n}\right) & =P^{n}\left(A^{n} \cap\left\{Z^{n, 2^{-N}}<\mu_{N}\right\}\right)+P^{n}\left(A^{n} \cap\left\{Z^{n, 2^{-N}} \geq \mu_{N}\right\}\right) \\
& <2^{-N}+P^{n}\left(A^{n} \cap\left\{Z^{n} \geq 2^{-N} \mu_{N}\right\}\right)<2^{-N}+\frac{2^{N}}{\mu_{N}} Q^{n}\left(A^{n}\right)<\gamma .
\end{aligned}
$$

\section{NAFL, NFL AND ORLICZ SPACES}

In Section 3 we mentioned that NAFL is a generalization of the concept NFL of Kreps [16] which was recalled in (2.1). We introduced Mackey-neighbourhoods of 0 to be able to describe an approximation with respect to the Mackey-topology for a sequence of $L^{\infty}$ spaces. Let us now take another look at NFL using the theory of Orlicz spaces. Although we have not yet mentioned the connection to Orlicz spaces, we already implicitly used them above by introducing the sets $B^{F}$. We will present now an alternative sight that enables us to replace the Mackey-neighborhoods by an approximation using norm-topologies (of the Orlicz spaces $L_{F}$ ). For each $F \in \mathcal{N}$ let

$$
L_{F}(P)=\left\{f \in L^{0}(P): E[F(a|f|)]<\infty \text { for some } a>0\right\} .
$$

We use the notation $L_{F}$ if it is clear which measure $P$ is meant. The so-called gauge norm on $L_{F}$ is given by

$$
\|f\|_{F}=\inf \left\{a>0: E\left[F\left(\frac{1}{a}|f|\right)\right] \leq 1\right\} .
$$

The space $L_{F}$ is called Orlicz space and it is well known that it is a Banach space with respect to $\|.\|_{F}$, see for example [19]. The set $B^{F}(P)$ defined in (3.2) and used in the proof of Theorem 3.3 is in fact the closed unit ball of $L^{F}(P)$. Indeed, it is easy to see that

$$
B^{F}(P)=\left\{f \in L_{F}(P):\|f\|_{F} \leq 1\right\}
$$


We will need a few facts on Orlicz spaces with respect to $N$-functions. We refer to [19] for all facts on Orlicz spaces. For $F \in \mathcal{N}$ there is a complementary $N$-function given by

$$
G(y)=\sup _{x \geq 0}(x y-F(x))
$$

The complementary of $G$ is again $F$. For each $F \in \mathcal{N}$ let

$$
L_{F}^{0}(P)=\left\{f \in L^{0}(P): E_{P}[F(a|f|)]<\infty \text { for all } a>0\right\} .
$$

For $F \in \mathcal{N}$ the space $L_{F}^{0}$ is the closed linear span of $L^{\infty}$ in $\left(L^{F},\|\cdot\|_{F}\right)$. Moreover the following holds.

Proposition 5.1. Let $(F, G)$ be a complementary pair of $N$-functions.

i) $E[|f g|] \leq 2\|f\|_{F}\|g\|_{G}$ for $f \in L_{F}$ and $g \in L_{G}$.

ii) If $g \in L_{G}$, then $\Phi: L_{F}^{0} \rightarrow \mathbb{R}$ given by $\Phi(f)=E[f g]$ is a continuous linear functional and

$$
\|g\|_{G} \leq\|\Phi\|_{\left(L_{F}^{0}\right)^{*}} \leq 2\|g\|_{G}
$$

iii) $L^{\infty}=\bigcap_{F \in \mathcal{N}} L_{F}$ and $L^{1}=\bigcup_{F \in \mathcal{N}} L_{F}$.

Recall that $V^{F}(P)=\left(B^{F}(P)\right)^{\circ}$. The following relation between the sets $V^{F}$ and $B^{G}$ holds.

Lemma 5.2. Let $(F, G)$ be a complementary pair of $N$-functions. Then

$$
\frac{1}{2} B^{G} \cap L^{\infty} \subseteq V^{F} \subseteq B^{G} \cap L^{\infty} .
$$

Proof. By Proposition 5.1 i) the first inclusion is clear. Let now $g \in V^{F}$. As $g \in L^{\infty}$ it is clear that $g$ defines a continuous linear functional $\Phi$ on $L_{F}^{0}$. As $|E[g h]| \leq 1$ for all $h \in B^{F}$, we have that $\|\Phi\|_{\left(L_{F}^{0}\right)^{*}} \leq 1$. Proposition 5.1 ii) implies that $\|g\|_{G} \leq\|\Phi\|_{\left(L_{F}^{0}\right)^{*}} \leq 1$. Hence $g \in B^{G} \cap L^{\infty}$.

We will use the following characterization of the closure with respect to the Mackey-topology of a convex subset $A$ of $L^{\infty}$. A very similar result can be found in Kusuoka [17]. We use the notation $\bar{A}^{F}$ for the closure of $A$ in $\left(L^{F},\|\cdot\|_{F}\right)$.

Lemma 5.3. Let $A$ be a convex subset of $L^{\infty}$. Then $\bigcap_{F \in \mathcal{N}} \bar{A}^{F}=\bar{A}^{\tau}$, where $\bar{A}^{\tau}$ is the closure of $A$ in $L^{\infty}$ with respect to the Mackey topology. (Note that, by convexity, $\bar{A}^{\tau}=\bar{A}^{*}$.)

Proof. It is clear that $\mathbf{A}:=\bigcap_{F \in \mathcal{N}} \bar{A}^{F}$ is a subset of $L^{\infty}$ by Proposition 5.1, (iii). We show that $\bigcap_{F \in \mathcal{N}} \bar{A}^{F}$ is closed with respect to the Mackey topology. Indeed, let $f \in \overline{\mathbf{A}}^{\tau}$. We will show that $f \in \mathbf{A}$. Fix an arbitrary N-function $G$. We have to show that $f \in \bar{A}^{G}$. Define, for any $\varepsilon>0, G_{\varepsilon}(x)=G\left(\frac{1}{\varepsilon} x\right)$ and let $F_{\varepsilon}$ be the complementary N-function of $G_{\varepsilon}$. As $f \in \overline{\mathbf{A}}^{\tau}$ and the sets $V^{F}$ are Mackeyneighborhoods we have that $f \in \mathbf{A}+V^{F}$ for all $F \in \mathcal{N}$, and so, in particular, $f \in \mathbf{A}+V^{F_{\varepsilon}}$ for any $\varepsilon>0$. Hence there exist $f_{\varepsilon} \in \mathbf{A}$ and $h_{\varepsilon} \in V^{F_{\varepsilon}}$ such that $f=f_{\varepsilon}+h_{\varepsilon}$. By Lemma 5.2 we have that $V^{F_{\varepsilon}} \subseteq B^{G_{\varepsilon}} \cap L^{\infty}$. By the definition of $G_{\varepsilon}$ moreover $B^{G_{\varepsilon}}=\varepsilon B^{G}$, and so $\left\|h_{\varepsilon}\right\|_{G} \leq \varepsilon$. As $f_{\varepsilon} \in \mathbf{A}$ we have that, in particular, $f_{\varepsilon} \in \bar{A}^{G}$ and so there exists $\tilde{f}_{\varepsilon} \in A$ such that $\left\|f_{\varepsilon}-\tilde{f}_{\varepsilon}\right\|_{G} \leq \varepsilon$. Hence we get that

$$
\left\|f-\tilde{f}_{\varepsilon}\right\|_{G} \leq\left\|f_{\varepsilon}-\tilde{f}_{\varepsilon}\right\|_{G}+\left\|h_{\varepsilon}\right\|_{G} \leq 2 \varepsilon
$$


As $\tilde{f}_{\varepsilon} \in A$ and as this can be done for any $\varepsilon>0$ we get that $f \in \bar{A}^{G}$. This works for any $G \in \mathcal{N}$ and so $f \in \mathbf{A}$, hence $\mathbf{A}$ is Mackey-closed.

Kusuoka [17] showed that $\bar{A}^{\tau}=\left(\bigcap_{F \in \mathcal{N}} C l_{F}(A)\right) \cap L^{\infty}$, where $C l_{F}(A)$ consists of all $f \in L_{F}^{0}$ such that there is a sequence $f^{n} \in A$ with $f^{n} \rightarrow f$ in probability and $\sup _{n} E\left[F\left(a\left|f^{n}\right|\right)\right]<\infty$ for all $a>0$. It is straightforward that $\bar{A}^{F} \subseteq C l_{F}(A)$. This concludes the proof.

\section{A formulation of NAFL in terms of Orlicz norms}

First we will reformulate the NFL-condition of (2.1).

Lemma 5.4. NFL holds if and only if for any $\varepsilon>0$

$$
\left(\bigcap_{F \in \mathcal{N}} \overline{\mathbf{C}}^{F}\right) \cap D^{\varepsilon}=\emptyset .
$$

Proof. Suppose there is NFL. Assume that there is $\alpha>0$ such that there exists $w \in\left(\bigcap_{F \in \mathcal{N}} \overline{\mathbf{C}}^{F}\right) \cap D^{\alpha}$. By Lemma 5.3 this is a contradiction, as $\bigcap_{F \in \mathcal{N}} \overline{\mathbf{C}}^{F}=\overline{\mathbf{C}}^{*}$. Now assume that the condition of the Lemma holds and that there is $w \in \overline{\mathbf{C}}^{*} \cap L_{+}^{\infty}$ and $w \not \equiv 0$. As $\mathbf{C}$ is a cone we have that $\tilde{w}:=\frac{w}{\|w\|_{\infty}} \in \overline{\mathbf{C}}^{*}$ as well. It is clear that there is $\alpha>0$ such that $\tilde{w} \in D^{\alpha}$. By Lemma 5.3 this is a contradiction.

The formulation of NFL as in Lemma 5.4 gives rise to the following alternative definition of no asymptotic free lunch using Orlicz norms. We denote the distance in $L^{F}(P)$ with respect to the norm by $\operatorname{dist}_{F}$.

Definition 5.5. We say that the large financial market satistfies the NAFL' condition if for any $\varepsilon>0$ there exist $F \in \mathcal{N}$ and $\delta>0$ such that for all $n \in \mathbb{N}$

$$
\operatorname{dist}_{F}\left(\mathbf{C}^{n}, D^{\varepsilon, n}\right) \geq \delta .
$$

The notion NAFL' says that it is not possible to approximate a strictly positive gain in all Orlicz norms. The advantage to Definition 3.2 is that we do not use the Mackey-topology which is not metrizable and therefore more technical than norm topologies. It turns out that the condition NAFL' is indeed equivalent to the condition NAFL. The proof is straightforward with the help of Lemma 5.2.

Proposition 5.6. $N A F L^{\prime} \Longleftrightarrow N A F L$.

Proof. Assume that NAFL does not hold. Fix an arbitrary $F \in \mathcal{N}$ and $\varepsilon>0$. Let $G$ be the complementary $N$-function of $F$ and define $G_{\varepsilon}(x)=G(\varepsilon x)$. This implies that $V^{G_{\varepsilon}, n}=\varepsilon V^{G, n}$ and so, by Lemma 5.2, $V^{G_{\varepsilon}, n} \subseteq \varepsilon\left(B^{F, n} \cap L^{\infty}\left(P^{n}\right)\right)$. By assumption there is $\alpha>0$ (which does not depend on $F$ or $\varepsilon$ ) such that for $G_{\varepsilon}$ there exist $n_{\varepsilon}$ and $f^{\varepsilon} \in \mathbf{C}^{n_{\varepsilon}}$ with $f^{\varepsilon}=w^{\varepsilon}+g^{\varepsilon}$, where $w^{\varepsilon} \in D^{\alpha, n_{\varepsilon}}$ and $g^{\varepsilon} \in V^{G_{\varepsilon}, n_{\varepsilon}}$. Therefore we have that

$$
\operatorname{dist}_{F}\left(D^{\alpha, n_{\varepsilon}}, \mathbf{C}^{n_{\varepsilon}}\right) \leq\left\|g^{\varepsilon}\right\|_{F} \leq \varepsilon,
$$

hence NAFL' does not hold.

Assume now that NAFL' does not hold. Let $F \in \mathcal{N}$ and $G$ be the complementary function of $F$. By assumption there is $\alpha>0$ (which does not depend on $F$ ), such that for any $\varepsilon>0$ there is $n_{\varepsilon}, f \in \mathbf{C}^{n_{\varepsilon}}$ and $w \in D^{\alpha, n_{\varepsilon}}$ such that $\|f-w\|_{G} \leq \varepsilon$. For $\varepsilon=1$ this gives that $g \in B^{G, n_{1}} \cap L^{\infty}\left(P^{n_{1}}\right) \subseteq V^{F, n_{1}}$, where $g:=f-w$. Hence NAFL does not hold. 


\section{REFERENCES}

[1] F. Delbaen and W. Schachermayer. A general version of the fundamental theorem of asset pricing. Math. Ann., 300:463-520, 1994.

[2] F. Delbaen and W. Schachermayer. The fundamental theorem of asset pricing for unbounded stochastic processes. Math. Ann., 312:215-250, 1998.

[3] M. Frittelli. Some remarks on arbitrage and preferences in securities market models. Math. Finance, 14:351-357, 2004

[4] J. Horvath. Topological Vector Spaces and Distributions. Addison-Wesley, 1966.

[5] G. Huberman. A simple approach to arbitrage pricing theory. J. Econom. Theory, 28:183-191, 1982.

[6] J. Jacod and A. N. Shiryaev. Limit Theorems for Stochastic Processes. Springer, 1987.

[7] Y. Kabanov. On the FTAP of Kreps-Delbaen-Schachermayer. In Statistics and Control of Stochastic Processes, the Lipster Festschrift (Y. Kabanov, B- L. Rozovskii and A.N. Shiryaev, eds.), World Scientific, Singapore, pages 191-203, 1997.

[8] Y. Kabanov Arbitrage Theory In Handbooks in Mathematical Finance. Option Procing: Theory and Practice, Cambridge University Press, pages 3-42, 2001.

[9] Y. Kabanov and D. Kramkov. Large financial markets: asymptotic arbitrage and contiguity. Theory Probab. Appl., 39: 222-228, 1994.

[10] Y. Kabanov and D. Kramkov. Asymptotic arbitrage in large financial markets. Finance and Stochastics, 2:143-172, 1998.

[11] I. Klein. A fundamental theorem of asset pricing for large financial markets. Math. Finance, 10:443-458, 2000.

[12] I. Klein. Market free lunch and large financial markets. to appear in Ann. Appl. Probab.

[13] I. Klein and W. Schachermayer Asymptotic arbitrage in non-complete large financial markets. Theory Probab. Appl., 41:927-934, 1996.

[14] I. Klein and W. Schachermayer A quantitative and a dual version of the Halmos-Savage theorem with applications to mathematical finance. Ann. Probab., 24:867-881, 1996.

[15] M. A. Krasnosel'skii and Y. B. Rutickii. Convex Functions and Orlicz Spaces. Gordon and Breach, 1961.

[16] D. M. Kreps. Arbitrage and equilibrium in economies with infinitely many commodities. $J$. Math. Econom., 8:15-35, 1981.

[17] S. Kusuoka. A remark on arbitrage and martingale measure. Publ. Res. Inst. Math. Sci., 29:833-840, 1993.

[18] J. Mémin. Espaces de semimartingales et changement de probabilité. Z. Wahrsch. Verw. Gebiete., 52:9-39, 1980.

[19] M. M. Rao and Z. D. Ren. Theory of Orlicz Spaces. Dekker, 1991.

[20] S. A. Ross. The arbitrage theory of asset pricing. J. Econom. Theory, 13:341-360, 1976

[21] P. Wojtaszczyk. Banach spaces for analysts. Cambridge University Press, 1991.

Dept. of Statistics and Decision Support Systems, University of Vienna, Brünnerstr. 72, A-1210 Vienna, Austria

E-mail address: Irene.Klein@univie.ac.at 